\title{
ON A GENERALIZATION OF THE CONLEY INDEX
}

\author{
MARIAN MROZEK \\ Institute of Computer Science, Jagiellonian University \\ 30-072 Kraków, Poland \\ E-mail:mrozek@ii.uj.edu.pl \\ JAMES F. REINECK \\ Department of Mathematics, SUNY at Buffalo \\ Buffalo, New York 14214-3093, U.S.A. \\ E-mail: reineck@newton.math.buffalo.edu \\ ROMAN SRZEDNICKI \\ Institute of Mathematics, Jagiellonian University \\ 30-059 Kraków, Poland \\ E-mail: srzednic@im.uj.edu.pl
}

1. Introduction. In this note we present the main ideas of the theory of the Conley index over a base space introduced in the papers $[7,8]$. The theory arised as an attempt to solve two questions concerning the classical Conley index. In the definition of the index, the exit set of an isolating neighborhood is collapsed to a point. Some information is lost on this collapse. In particular, topological information about how a set sits in the phase space is lost. The first question addressed is how to retain some of the information which is lost on collapse. As an example, is it possible that two repelling periodic orbits which are not homotopic in the punctured plane are related by continuation? Clearly one cannot be continued to the other as periodic orbits, but the index of such a periodic orbit is the same as the index of the disjoint union of two rest points, so the question of continuation as isolated invariant sets is far less obvious.

To solve this problem, we fix a continuous map $\xi: X \rightarrow Z$ from the phase space $X$ to some Hausdorff space $Z$ (called the base space). Let $S$ be an isolated invariant set in

1991 Mathematics Subject Classification: 34C35, 54H20.

Research of M. Mrozek supported by KBN, Grant 2 P03A 02912.

Research of R. Srzednicki supported by KBN, Grant 2 P03A 04010.

The paper is in final form and no version of it will be published elsewhere. 
$X$. For a regular index pair $\left(P_{1}, P_{2}\right)$ for $S$ the adjunction $P_{1} \cup_{\left.\xi\right|_{P_{2}}} Z$ is obtained by gluing the exit set $P_{2}$ to $Z$ via the map $\xi$. The so-called fiberwise deforming homotopy type of the adjunction is independent of the choice of regular index pair for $S$, and is called the Conley index over the base space $Z$ (more precisely: with the base map $\xi$ ). The definition of fiberwise deforming homotopy type is given below, but it is a finer invariant than the usual homotopy type of the adjunction. If $Z$ is a point, then this gives the classical Conley index. Another possibility for an arbitrary flow is $Z=X, \xi=\mathrm{id}_{X}$. These two examples represent the maximal and minimal information loss due to collapsing. Other spaces $Z$ and maps $\xi$ lie somewhere in between in terms of information lost upon collapse.

The index over a base has properties similar to the classical Conley index. It is additive, multiplicative, and invariant under continuation. The construction of the index over a base is fully functorial. In particular, this means that the new index extends to a connected simple system, and one may also obtain a Conley functor as in [4]. By making a judicious choice of $Z$ and $\xi$, one can often obtain more information than in the classical case.

In the case of periodic orbits in different homotopy classes, let $X$ denote the punctured plane $\mathbb{R}^{2} \backslash\{0\}, Z$ be the unit circle, and $\xi(x)=x /|x|$. Suppose $S$ is a repelling periodic orbit, with $P_{1}$ an annulus, and $P_{2}=\partial P_{1}$. If $S$ is homotopically nontrivial, then the index of $S$ over $Z$ is a 2-torus, but if $S$ is homotopically trivial, it is the wedge of a 2 -sphere and two 1 -spheres. Thus these sets are not related by continuation in any family of flows on the punctured plane. If the periodic orbits are attracting, then the resulting adjunction space is homotopically two circles regardless of the homotopy class of the periodic orbit. However, the fiberwise deforming homotopy type of the sets are different if the periodic orbits are in different homotopy classes.

A Poincaré section is a frequently used tool in the study of dynamical systems. It lets one translate certain problems in the continuous dynamics of flows to their counterparts in the discrete dynamics of the associated Poincaré map. One of the advantages of such an approach is that the dimension of phase space is reduced by one. The main disadvantage lies in the fact that often very little is known about the Poincaré map beyond its existence. The development of the discrete counterpart of the Conley index (see $[10,6,11]$ ) raised hopes that the interaction between the dynamics of a flow with a Poincaré section and the dynamics of the Poincaré map may be fruitfully observed and applied on the level of the Conley index theory. It is fairly easy to show that the cohomological Conley index of the Poincaré map completely determines the cohomological Conley index of the flow (see [5]). The second question addressed in this note is the reverse problem: is it possible to reconstruct the Conley index for the Poincaré map from the Conley index for flow? Unfortunately it turns out that such a reconstruction is not possible with the classical Conley index as simple examples show (see [8]). However the Conley index over the circle carries all the information necessary to compute the Conley index of the Poincaré map. Since the computation of the Conley index over the circle requires, as in the case of the classical Conley index for flows, only the local knowledge of the flow, replacing the classical Conley index by the Conley index over the circle seems to solve completely the problem. 
Let $N$ be an isolating neighborhood in a semiflow $\pi$, which admits $\Sigma$, a Poincaré section for $N$. Then there is an associated discrete dynamical system, namely the Poincaré map $\Pi$ on $\Sigma$. If $S$ denotes the maximal invariant set in $N$, then $K:=S \cap \Sigma$ is an isolated invariant set for $\Pi$. To obtain the discrete Conley index of $K$ with respect to $\Pi$ from looking at the flow in $N$ we first consider the index of $S$ over the base $\mathbb{S}^{1}$. The adjunction $\left.P_{1} \cup \xi\right|_{P_{2}} \mathbb{S}^{1}$ is a fiberwise pointed space over $\mathbb{S}^{1}$ and it carries an additional structure similar in spirit to a locally trivial bundle. We call this the semibundle structure. Roughly speaking, any $\xi$-preserving semiflow on $P_{1} \cup_{\left.\xi\right|_{P_{2}}} \mathbb{S}^{1}$ defines the same semibundle structure. The homotopy class of the map induced by the semiflow on every fiber leads, via the universal functor defined by Szymczak (see [11]), to an invariant of fiberwise deforming homotopy types of semibundles called the monodromy class. All this makes the Conley index over the circle an invariant of isolating neighborhoods which is much stronger than the classical Conley index. An important manifestation of this fact is Theorem 6.7. It states that the Conley index of $K$ with respect to the Poincare map $\Pi$ is completely determined by the Conley index of $S$ over $\mathbb{S}^{1}$, namely it is the monodromy class of $h_{\xi}(S)$.

We want to emphasize that the Conley index over the circle in principle is not more difficult to compute than the classical Conley index. Moreover, although one way to find the semibundle structure on $P_{1} \cup_{\left.\xi\right|_{P_{2}}} \mathbb{S}^{1}$ is via the semiflow induced from the original flow, any other $\xi$-preserving semiflow on $P_{1} \cup_{\xi \mid P_{2}} \mathbb{S}^{1}$ defines the same semibundle structure. Therefore in practice only the knowledge of the topology of a regular index pair and the map $\xi$ is needed to compute both the index over $\mathbb{S}^{1}$ and its monodromy class.

At least one important question remains unsolved. By using the extension of the classical index to the index over $\mathbb{S}^{1}$, we are able to determine the index for the Poincaré map. However, we do not know if the correspondence is 1-1, i.e. if different indices over $\mathbb{S}^{1}$ necessarily give different indices for the Poincaré map.

To keep things as simple as possible in the present paper we restricted our attention to $\xi$-preserving flows. This is a satisfactory setting for ODE's with periodic forcing but things become technically significantly more complicated if one wants to consider an autonomous differential equation with a Poincaré section. Therefore the machinery needed in that case will be presented in a future paper.

We denote the sets of natural numbers by $\mathbb{N}$, the set of all integers by $\mathbb{Z}$ and the set of all real numbers by $\mathbb{R}$. For a given topological space $X$ and $A \subset X$, we denote the closure of $A$ by cl $A$, its interior by int $A$ and its boundary by bd $A$. The unit circle in the complex plane is denoted by $\mathbb{S}^{1}$.

2. Fiberwise pointed spaces over a base. Let $Z$ be a fixed topological space. A triple $\mathbf{U}=(U, r, s)$ is called a fiberwise pointed (topological) space over $Z$ if $U$ is a topological space, $r: U \rightarrow Z$ and $s: Z \rightarrow U$ are continuous maps, and $r \circ s=\operatorname{id}_{Z}$ (compare [3, p. 41-42]). In [1, p. 163] and [2, p. 98] such a space is also called a sectioned space over $Z$. We refer to $Z$ as the base space, to $r$ as the projection, and to $s$ as the section of $\mathbf{U}$. The projection is an r-map in the sense of [Bo]. In particular it follows that $s(Z)$ is a retract (hence a closed subset) of $U, s \circ r: U \rightarrow s(Z)$ is a retraction, and $s$ and the restriction of $r$ are mutually inverse homeomorphisms between $Z$ and $s(Z)$. 
We want to make fiberwise pointed spaces into a category. Let $\mathbf{U}^{\prime}=\left(U^{\prime}, r^{\prime}, s^{\prime}\right)$ be another fiberwise pointed space over $Z$. A natural choice for a morphism from $\mathbf{U}$ to $\mathbf{U}^{\prime}$ is to take a fiberwise pointed map, i.e. a continuous map $\phi: U \rightarrow U^{\prime}$ such that $r^{\prime} \circ \phi=r$ and $\phi \circ s=s^{\prime}$ (comp. [1,3] for instance). For our purposes such a definition is too restrictive.

We define a fiberwise deforming map (briefly: an f.d. map) $f: \mathbf{U} \rightarrow \mathbf{U}^{\prime}$ as a continuous map $f:(U, s(Z)) \rightarrow\left(U^{\prime}, s^{\prime}(Z)\right)$ such that

$$
r^{\prime} \circ f \simeq r \operatorname{rel} s(Z)
$$

Obviously a fiberwise pointed map is an f.d. map. Note that there always exists a fiberwise pointed map (and consequently an f.d. map) between $\mathbf{U}$ and $\mathbf{U}^{\prime}$, namely $s^{\prime} \circ r$ (it is called the fiberwise constant map).

Proposition 2.1. If $f: \mathbf{U} \rightarrow \mathbf{U}^{\prime}$ is $f$.d. then $f \circ s=s^{\prime}$ and the restriction of $f$ to a map $s(Z) \rightarrow s^{\prime}(Z)$ is equal to the homeomorphism $\left.s^{\prime} \circ r\right|_{s(Z)}$. In particular $f(s(Z))=$ $s^{\prime}(Z)$.

One can easily verify that the identity map is an f.d. map and the composition of f.d. maps is an f.d. map. Thus fiberwise pointed spaces over $Z$ together with f.d. maps constitute a category. We will call it the fiberwise deforming category and we will denote it by $\mathrm{Fib}_{Z}$.

One can define the wedge and smash products of fiberwise pointed spaces.

By a homotopy in the category $\mathrm{Fib}_{Z}$ we mean a collection of f.d. maps $F_{t}: \mathbf{U} \rightarrow$ $\mathbf{U}^{\prime}, t \in I$ such that the map $F: U \times I \ni(x, t) \rightarrow F_{t}(x) \in U^{\prime}$ is continuous. We say that two f.d. maps $f, g: \mathbf{U} \rightarrow \mathbf{U}^{\prime}$ are homotopic in $\mathrm{Fib}_{Z}$ if there exists a homotopy $F_{t}: \mathbf{U} \rightarrow \mathbf{U}^{\prime}, t \in I$ in $\mathrm{Fib}_{Z}$ such that $F_{0}=f, F_{1}=g$.

As an easy consequence of Proposition 2.1 we obtain the following If

Proposition 2.2. Assume $f: \mathbf{U} \rightarrow \mathbf{U}^{\prime}$ is an f.d. map and $g: U \rightarrow U^{\prime}$ is continuous.

$$
f \simeq g \operatorname{rel} s(Z)
$$

then $g$ is also an f.d. map from $\mathbf{U}$ to $\mathbf{U}^{\prime}$ and $f$ and $g$ are homotopic in $\mathrm{Fib}_{Z}$.

The proof of this result, and the others in this section, can be found in [7].

Obviously, the homotopy in the category $\mathrm{Fib}_{Z}$ induces an equivalence relation between the f.d. maps. Taking equivalence classes of f.d. maps as a new collection of morphisms we obtain another category, which we will call the fiberwise deforming homotopy category. We will denote it by $\mathrm{HtpFib}_{Z}$. Obviously we have also the homotopy functor Htp : Fib $\rightarrow$ $\mathrm{HtpFib}_{Z}$ sending each f.d. map to its equivalence class. An f.d. map $\phi$ will be called a fiberwise deforming homotopy equivalence if $\operatorname{Htp}(\phi)$ is an isomorphism in $\operatorname{HtpFib}_{Z}$.

Proposition 2.3. Assume $f: \mathbf{U} \rightarrow \mathbf{U}^{\prime}$ is an f.d. map. If there exists a continuous map $f^{\prime}: U^{\prime} \rightarrow U$ such that

$$
f^{\prime} \circ f \simeq \operatorname{id}_{U} \operatorname{rel} s(Z), f \circ f^{\prime} \simeq \operatorname{id}_{U^{\prime}} \operatorname{rel} s^{\prime}(Z)
$$

then $f^{\prime}$ is also an f.d. map and $f$ and $f^{\prime}$ are mutually inverse fiberwise deforming homotopy equivalences. 
We say that two fiberwise pointed spaces $\mathbf{U}$ and $\mathbf{U}^{\prime}$ over $Z$ have the same fiberwise deforming homotopy type over $Z$ if there exists a fiberwise deforming homotopy equivalence between them. The equivalence class of spaces with the same fiberwise deforming homotopy type as $\mathbf{U}$ is denoted by $[\mathbf{U}]_{Z}$. A distinguished role is played by

$$
\overline{0}_{Z}:=\left[\left(Z, \mathrm{id}_{Z}, \mathrm{id}_{Z}\right)\right]_{Z}
$$

the trivial fiberwise deforming homotopy type over $Z$.

We compare various homotopy types on a simple example.

EXAmple 2.4. For $x \in \mathbb{R} \backslash 0$ put $V_{x}=(\mathbb{R} \backslash 0) \times 0 \cup\{x\} \times 1$, i.e. $V_{x}$ is the disjoint union of $\mathbb{R} \backslash 0$ and $\{x\}$. Define $r_{x}: V_{x} \rightarrow \mathbb{R} \backslash 0$ and $s_{x}: \mathbb{R} \backslash 0 \rightarrow V_{x}$ by $r_{x}(y, i)=y$ and $s_{x}(z)=(z, 0)$. Then $\mathbf{V}_{x}=\left(V_{x}, r_{x}, s_{x}\right)$ is a fiberwise pointed space over $\mathbb{R} \backslash 0$. Each of the spaces $V_{x}$ has the same (ordinary) homotopy type of a three-point space. It is easy to see, that for $x \neq x^{\prime}$ there is no fiberwise pointed map $\mathbf{V}_{x} \rightarrow \mathbf{V}_{x^{\prime}}$ except of the constant one, hence the fiberwise pointed homotopy types (i.e. the homotopy types in the sense of $[1,3])$ of $\mathbf{V}_{x}$ are all different. On the other hand there are no nonconstant f.d. maps between $\mathbf{V}_{x}$ and $\mathbf{V}_{x^{\prime}}$ only in the case where the signs of $x$ and $x^{\prime}$ are opposite, hence there are exactly two different fiberwise deforming homotopy types, and $\left[\mathbf{V}_{x}\right]_{\mathbb{R} \backslash 0}=\left[\mathbf{V}_{x^{\prime}}\right]_{\mathbb{R} \backslash 0}$ iff $x x^{\prime}>0$.

In the above example we compared the fiberwise pointed homotopy types of spaces with several connected components. This can be generalized to the following simple observation.

Proposition 2.5. Let $\left\{T_{i}\right\}_{i=0, \ldots, r}$ and $\left\{T_{i}^{\prime}\right\}_{i=0, \ldots, r}$ be the sets of path components of $U$ and $U^{\prime}$, respectively. Let

$$
s(Z) \subset T_{0}, s^{\prime}(Z) \subset T_{0}^{\prime} .
$$

If $f: \mathbf{U} \rightarrow \mathbf{U}^{\prime}$ is a fiberwise deforming homotopy equivalence then there is a permutation $\sigma, \sigma(0)=0$, such that for $i=0, \ldots, r: f\left(T_{i}\right) \subset T_{\sigma(i)}^{\prime}$ and the restriction $f_{i}: T_{i} \rightarrow T_{\sigma(i)}$ of $f$ is a homotopy equivalence such that $\left.r^{\prime} \circ f_{i} \simeq r\right|_{T_{i}}$

In order to distinguish fiberwise deforming homotopy types of $\mathbf{U}$ and $\mathbf{U}^{\prime}$ one should compare the homotopy types $[U]$ and $\left[U^{\prime}\right]$ first. If they are equal, further necessary information can be provided by Proposition 2.5 as the next example shows:

EXAMPLE 2.6. Let $W_{1}=W_{2}=S^{1} \times 0 \cup S^{1} \times 1$, i.e. $W_{1}$ and $W_{2}$ are equal to the disjoint union of two copies of the circle $S^{1}=\{x \in \mathbb{C}:|x|=1\}$. Define $r_{i}: W_{i} \rightarrow S^{1}$ and $s_{i}: S^{1} \rightarrow W_{i}, i=1,2$ by

$$
\begin{gathered}
r_{1}(x, i)=x, s_{1}(y)=(y, 0), \\
r_{2}(x, 0)=x, r_{2}(x, 1)=x^{2}, s_{2}(y)=(y, 0) .
\end{gathered}
$$

Then $\mathbf{W}_{i}=\left(W_{i}, r_{i}, s_{i}\right), i=1,2$ are fiberwise pointed spaces over $S^{1}$. It follows by Proposition 2.5 that if $f: \mathbf{W}_{1} \rightarrow \mathbf{W}_{2}$ is a fiberwise pointed homotopy equivalence then $f\left(S^{1} \times 1\right) \subset\left(S^{1} \times 1\right)$ and $\left.\left.r_{2} \circ f\right|_{S^{1} \times 1} \simeq r_{1}\right|_{S^{1} \times 1}$, which is impossible. Thus $\left[\mathbf{W}_{1}\right]_{S^{1}} \neq$ $\left[\mathbf{W}_{2}\right]_{S^{1}}$ 
It is easily seen that if $[\mathbf{U}]_{Z}=\left[\mathbf{U}^{\prime}\right]_{Z}$ and $[\mathbf{V}]_{Z}=\left[\mathbf{V}^{\prime}\right]_{Z}$ then $[\mathbf{U} \vee \mathbf{V}]_{Z}=\left[\mathbf{U}^{\prime} \vee \mathbf{V}^{\prime}\right]_{Z}$, hence we can define

$$
[\mathbf{U}]_{Z} \vee[\mathbf{V}]_{Z}:=[\mathbf{U} \vee \mathbf{V}]_{Z}
$$

the wedge of fiberwise deforming homotopy types. Obviously, for every space $\mathbf{U}$,

$$
[\mathbf{U}]_{Z} \vee \overline{0}_{Z}=\overline{0}_{Z} \vee[\mathbf{U}]_{Z}=[\mathbf{U}]_{Z}
$$

Some additional assumptions are needed to show that the smash products of fiberwise deforming homotopy types is well defined (e.g. $s$ is a cofibration). Details are presented in $[7]$.

3. The Conley index over a base. Given an arbitrary set $A \subset X$, we define the exit function on $A$ by

$$
\epsilon_{A}: A \ni x \longrightarrow \sup \{t \geq 0 \mid x \cdot[0, t] \subset A\} \in[0, \infty] .
$$

Let $\xi: X \rightarrow Z$ be a fixed continuous map. Let $S$ be an isolated invariant set in a semiflow on $X$, and let $P=\left(P_{1}, P_{2}\right)$ be an index pair for $S$. Define functions $\sigma_{P}, \tau_{P}$ : $P_{1} \rightarrow[0, \infty]$ by

$$
\begin{aligned}
\sigma_{P}(x) & := \begin{cases}\epsilon_{\mathrm{cl}\left(P_{1} \backslash P_{2}\right)}(x) & \text { if } x \in \operatorname{cl}\left(P_{1} \backslash P_{2}\right) \\
0 & \text { if } x \in \operatorname{int}_{P_{1}} P_{2}\end{cases} \\
\tau_{P}(x) & := \begin{cases}\sup \left\{t \geq 0 \mid x \cdot[0, t] \subset P_{1} \backslash P_{2}\right\} & \text { if } x \in P_{1} \backslash P_{2} \\
0 & \text { if } x \in P_{2}\end{cases}
\end{aligned}
$$

Recall that $P$ is called regular if $\tau_{P}(x)=\sigma_{P}(x)$ for $x \in P_{1}$. Let $B$ be a compact subset of $X$. If $\pi$ is a flow, we define

$$
\begin{array}{r}
B^{+}=:\left\{x \in B: \exists \epsilon_{n}>0, \epsilon_{n} \rightarrow 0: x .\left(-\epsilon_{n}\right) \notin B\right\}, \\
B^{-}=:\left\{x \in B: \exists \epsilon_{n}>0, \epsilon_{n} \rightarrow 0: x . \epsilon_{n} \notin B\right\} .
\end{array}
$$

We call $B$ an isolating block for the flow $\pi$ if both $B^{+}$and $B^{-}$are compact and $\partial B=$ $B^{+} \cup B^{-}$. If $B$ is an isolating block for $S$ and $B^{-}$is its exit set, then $\left(B, B^{-}\right)$is a regular index pair.

We recall (see [7]) that for $P=\left(P_{1}, P_{2}\right)$ a pair of compact spaces, $U_{\xi}(P)$ is defined as the adjunction $P_{1} \cup_{\left.\xi\right|_{P_{2}}} Z$, i.e.

$$
U_{\xi}(P):=\mathbb{S}^{1} \times 0 \cup P_{1} \times 1 / \sim,
$$

where $\sim$ denotes the minimal equivalence relation such that $(u, 1) \sim(\xi(u), 0)$ for every $u \in P_{2}$. For $(u, i) \in \mathbb{S}^{1} \times 0 \cup P_{1} \times 1$ denote by $[u, i]$ the equivalence class of $(u, i)$ in the relation $\sim$. One easily checks that

$$
\mathbf{U}_{\xi}(P):=\left(U_{\xi}(P), r_{U_{\xi}(P)}, s_{U_{\xi}(P)}\right),
$$

where

is a fiberwise pointed space.

$$
r_{U_{\xi}(P)}([x, i]):=\xi(x), \quad s_{U_{\xi}(P)}(a):=[a, 0],
$$

Theorem 3.1 ([7]). Let $S$ be an isolated invariant set. Then $h_{\xi}(S, \pi):=\left[\mathbf{U}_{\xi}(P)\right]_{Z}$ (i.e. the fiberwise deforming homotopy type of $\mathbf{U}_{\xi}(P)$ over $Z$ ) is independent of the choice of an isolating neighborhood $N$ of $S$ and of a regular index pair $P$ in $N$. 
The homotopy type $h_{\xi}(S, \pi)$ given by the above theorem (denoted briefly by $h_{\xi}(S)$ if $\pi$ is clear from the context) is called the Conley index of $S$ over base $Z$ with base map $\xi$ or just the Conley index of $S$ over $Z$ if $\xi$ is clear from context.

As in the classical case, $h_{\xi}(S) \neq \overline{0}_{Z}$ implies $S \neq \emptyset$, the index of the disjoint union of invariant sets is the wedge of the indices over $Z$, and the index of the Cartesian product of invariant sets is the smash product of the indices over $Z$.

Let $z_{0}$ be a point in $Z$ and let $c:=c_{z_{0}}: X \rightarrow Z, x \mapsto z_{0}$, be a constant map. If $P$ is a closed pair then $U_{c}(P)=P_{1} / P_{2} \vee\left(Z, z_{0}\right)$ and $r_{c, P}\left([x, 1]_{c, P}\right)=z_{0}$ for every $x \in P_{1}$. In that case the fiberwise deforming homotopy type $\left[\mathbf{U}_{c}(P)\right]_{Z}$ is called simple. In this case the index over $Z$ contains no more information than the classical index, at least if $Z$ is path connected. In particular, if $S$ sits in $X$ in a topologically trivial way, then the index over a base adds nothing to the classical index.

Proposition 3.2. If $P=\left(P_{1}, P_{2}\right)$ is cofibered and $P_{1}$ is contractible in $X$ then $\left[\mathbf{U}_{\omega}(P)\right]_{Z}$ is simple for every $\omega: X \rightarrow Z$.

Consider a family of semiflows $\pi^{\lambda}: X \times \mathbb{R}^{+} \rightarrow X$ continuously depending on $\lambda \in I$. We have the following continuation theorem which is analogous to the continuation result for the classical Conley index.

THEOREM 3.3. If $N$ is an isolating neighborhood with respect to $\pi^{\lambda}$ for all $\lambda \in I$ and $S^{\lambda}$ is the maximal invariant set of $\pi^{\lambda}$ inside $N$ then $h_{\xi}\left(S^{\lambda}, \pi^{\lambda}\right)$ does not depend on $\lambda$.

In the following examples the term "index" means $h_{\xi}(S)$ for a given isolated invariant set $S$. Here we use the simplest and most natural choices of $\xi$ and $Z$ to obtain information which is more delicate than that obtained by the classical index. We begin with our example from the introduction.

EXAMPLE 3.4. Suppose $X$ is the punctured plane, $\gamma_{1}$ is a homotopically nontrivial repelling periodic orbit, and $\gamma_{2}$ is a homotopically trivial repelling orbit. Let $Z$ be the unit circle and $\xi$ be the map $z \mapsto z /|z|$. By Proposition 3.2, the index of $\gamma_{2}$ is simple, i.e. has the fiberwise deforming homotopy type of the one-point wedge of the classical Conley index of $\gamma_{1}$ and $Z$. In this case the classical index is the wedge of a 1-sphere and a 2-sphere, hence the (usual) homotopy type of the index is that of $\mathbb{S}^{1} \vee \mathbb{S}^{1} \vee \mathbb{S}^{2}$. The index of $\gamma_{1}$ over $Z$ has the homotopy type of the 2 -torus $\mathbb{S}^{1} \times \mathbb{S}^{1}$. (This can be seen either by homotoping $Z$ to $\gamma_{1}$ and homotoping $\xi$ in a corresponding way, or by continuing $\gamma_{1}$ to a circle in the plane, and using an annulus as $P_{1}$.) Since the usual homotopy types of the indices of $\gamma_{1}$ and $\gamma_{2}$ over $Z$ are different, the fiberwise deforming homotopy types are also different, so by Theorem 3.3, they are not related by continuation.

The next example shows that fiberwise deforming homotopy type is a finer classification than ordinary homotopy type.

EXAmPle 3.5. Again, suppose $X$ is the punctured plane, $Z$ is the unit circle and $\xi$ is the map $z \mapsto z /|z|$, but this time the periodic orbits are attracting, with $\gamma_{1}$ homotopically nontrivial and $\gamma_{2}$ homotopically trivial. We use annuli as the isolating neighborhoods and as $P_{1}$ in the index pair. In this example, since the exit set $P_{2}$ is empty, both indices over $Z$ have the same usual homotopy type, namely the disjoint union of two circles, one coming 
from $P_{1}$, the other from $Z$. However, the projections $r_{1}$ and $r_{2}$, for $\gamma_{1}$ and $\gamma_{2}$ respectively, map the annulus into different homotopy classes in $Z$. It follows from Proposition 2.5 that the resulting adjunction spaces are not fiberwise deforming homotopy equivalent, so again by Theorem $3.3, \gamma_{1}$ and $\gamma_{2}$ are not related by continuation.

4. Semibundles over the circle. The rest of this paper is concerned with recovering the index of the Poincare map from the index over the circle. We base on the paper [8], to which we refer for subsequent omitted proofs.

Let $\pi: X \times \mathbb{R}^{+} \rightarrow X$ be a semiflow. Let $\pi_{t}:=\pi(\cdot, t)$ denote the $t$-translation operator of $\pi$. We fix the base to be the circle $\mathbb{S}^{1}$ and fix the map $\xi: X \rightarrow \mathbb{S}^{1}$. We say that $\pi$ is $\xi$-preserving if for all $x \in X$ and all $t \in \mathbb{R}^{+}$

$$
\xi\left(\pi_{t}(x)\right)=e^{2 \pi i t} \xi(x) .
$$

A fiberwise pointed space $\mathbf{U}=(U, r, s)$ over $\mathbb{S}^{1}$ is called a semibundle if there exists an $r$-preserving semiflow $\varphi: U \times \mathbb{R}^{+} \rightarrow U$ such that $\varphi(s(a), t)=s\left(e^{2 \pi i t} a\right)$ for any $a \in \mathbb{S}^{1}$ and $t \in \mathbb{R}^{+}$. Such a semiflow is called a semibundle carrier on $\mathbf{U}$. The name semibundle is justified by the fact that if $\mathbf{U}$ admits a semibundle carrier which is a flow then $r: U \rightarrow \mathbb{S}^{1}$ is a locally trivial bundle. Note that the semibundle carrier is not unique in general.

The 1-translation operator or the time-one map $\varphi_{1}$ of the semibundle carrier $\varphi$ on $\mathbf{U}$ plays a special role in the sequel. Let us denote it by $\Phi$, and denote its restriction to the fiber $U_{a}:=r^{-1}(a)$ over $a$ by $\Phi_{a}$.

Proposition 4.1. The time-one map $\Phi: \mathbf{U} \rightarrow \mathbf{U}$ is a fiberwise pointed map. For every $a \in \mathbb{S}^{1}$ the homotopy class of $\Phi_{a}$ in $\mathrm{Top}_{*}$ is independent of the choice of a semibundle carrier $\varphi$.

Proof. The first assertion is obvious. If $\varphi^{\prime}$ is another semibundle carrier on $\mathbf{U}$ then the required homotopy is $\varphi_{1-t}^{\prime} \varphi_{t}$.

5. The Szymczak category and discrete Conley index. Let $\mathcal{E}$ be a category. The category of endomorphisms of $\mathcal{E}$, denoted by $\operatorname{Endo}(\mathcal{E})$, is defined as follows. The objects of $\operatorname{Endo}(\mathcal{E})$ are pairs $(A, a)$, where $A \in \mathcal{E}$ and $a \in \mathcal{E}(A, A)$ is an endomorphism of $A$. The set of morphisms from $(A, a) \in \operatorname{Endo}(\mathcal{E})$ to $(B, b) \in \operatorname{Endo}(\mathcal{E})$ is the subset of $\mathcal{E}(A, B)$ consisting of exactly those morphisms $f \in \mathcal{E}(A, B)$ for which $b \circ f=f \circ a$. We write $f:(A, a) \rightarrow(B, b)$ to denote that $f$ is a morphism from $(A, a)$ to $(B, b)$ in $\operatorname{Endo}(\mathcal{E})$. Note that if $(A, a) \in \operatorname{Endo}(\mathcal{E})$ then $a \in \operatorname{Endo}(\mathcal{E})((A, a),(A, a))$.

The definition of the Szymczak category of $\mathcal{E}$ (see [11]), denoted by $\operatorname{Szym}(\mathcal{E})$, is slightly more complicated. The objects in $\operatorname{Szym}(\mathcal{E})$ coincide with the objects in $\operatorname{Endo}(\mathcal{E})$, i.e. the pairs $(A, a)$ such that $a$ is an endomorphism of $A$. To define morphisms we first introduce the following relation for $(f, m),(g, n) \in \operatorname{Endo}(\mathcal{E})((A, a),(B, b)) \times \mathbb{N}$ :

$$
(f, m) \equiv(g, n): \Longleftrightarrow \exists k \in \mathbb{N} f \circ a^{n+k}=g \circ a^{m+k} .
$$

One easily verifies that the relation is an equivalence relation and

$$
(f, m) \equiv(g, n): \Longleftrightarrow \exists k \in \mathbb{N} b^{n+k} \circ f=b^{m+k} \circ g .
$$


The morphisms in the Szymczak category are now defined as the equivalence classes of the relation $\equiv$ :

$$
\operatorname{Szym}(\mathcal{E})((A, a),(B, b)):=[\operatorname{Endo}(\mathcal{E})((A, a),(B, b)) \times \mathbb{N}] / \equiv .
$$

Let $[f, m] \in \operatorname{Szym}(\mathcal{E})((A, a),(B, b))$ and $[g, n] \in \operatorname{Szym}(\mathcal{E})((B, b),(C, c))$. It is straightforward to check that $\left[\operatorname{id}_{A}, 0\right]$ is the identity on $(A, a)$ in $\operatorname{Szym}(\mathcal{E})$ and $[g \circ f, m+n]$ is a well defined morphism in $\operatorname{Szym}(\mathcal{E})((A, a),(C, c))$, which by definition is the composition of morphisms $[f, m]$ and $[g, n]$ (denoted by $[g, n] \circ[f, m]$ ). There is a canonical functor from $\operatorname{Endo}(\mathcal{E})$ to $\operatorname{Szym}(\mathcal{E})$ which is equal to the identity on the objects of $\operatorname{Endo}(\mathcal{E})$ and which transforms every morphism $f$ in $\operatorname{Endo}(\mathcal{E})$ to $[f, 0]$ in $\operatorname{Szym}(\mathcal{E})$. We note for future reference the following straightforward proposition.

Proposition 5.1. If $(A, a)$ is an object in $\operatorname{Szym}(\mathcal{E})$ then

(a) $\left[a^{n}, n\right]=\left[\operatorname{id}_{A}, 0\right]$ for each $n \in \mathbb{N}$.

(b) $\left[a^{p}, q\right]$ is an isomorphism for every $p, q \in \mathbb{N}$; its inverse is equal to $\left[\mathrm{id}_{A}, p-q\right]$ if $p \geq q$ and $\left[a^{q-p}, 0\right]$ if $p \leq q$.

The reason to introduce the Szymczak category is the following theorem.

Theorem 5.2. Assume that $(A, a),(B, b) \in \operatorname{Endo}(\mathcal{E})$. Let $f$ and $g$ be morphisms in $\mathcal{E}$ such that the diagram

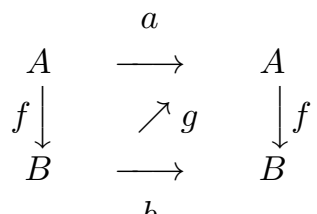

commutes. Then $f \in \operatorname{Endo}(\mathcal{E})((A, a),(B, b)), g \in \operatorname{Endo}(\mathcal{E})((B, b),(A, a))$ and we have the commutative diagram

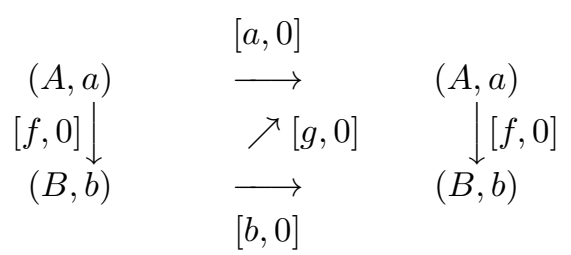

in $\operatorname{Szym}(\mathcal{E})$, in which all morphisms are isomorphisms.

Proof. By Proposition $5.1[a, 0]$ and $[b, 0]$ are isomorphisms. It is now a standard categorical argument to show that also $[f, 0]$ and $[g, 0]$ are isomorphisms.

For $A \in \mathcal{E}$ and $a \in \mathcal{E}(A, A)$ the equivalence class

$$
[A, a]_{\text {Szym }}:=\{(B, b) \mid(B, b) \text { is isomorphic to }(A, a) \text { in } \operatorname{Szym}(\mathcal{E})\}
$$

will be called the $S z y m c z a k$ class of $(A, a)$ in $\mathcal{E}$.

Now let $f: X \rightarrow X$ be a continuous map on a locally compact metric space $X$. Recall that in the discrete case (see [11], [6]) the pair $P=\left(P_{1}, P_{2}\right)$ of closed subsets of an isolating neighborhood $N$ will be called an index pair of $K$ in $N$ (with respect to $f$ ) 
if the following three conditions are satisfied.

$$
\begin{gathered}
P_{i} \cap f^{-1}(N) \subset f^{-1}\left(P_{i}\right) \text { for } i=0,1, \\
P_{1} \backslash P_{2} \subset f^{-1}(N), \\
K \subset \operatorname{int}\left(P_{1} \backslash P_{2}\right) .
\end{gathered}
$$

With every index pair $P$ one associates an object $\left(P_{*},\left[f_{P}\right]\right) \in \operatorname{Endo}\left(\mathrm{HtpTop}_{*}\right)$ as follows. $P_{*}$ is a pointed topological space $P_{1} / P_{2}$ with the distinguished point $\left[P_{2}\right]$ being the set $P_{2}$ collapsed to a point. The morphism $\left[f_{P}\right]$ is the pointed homotopy class of the induced map $f_{P}: P_{1} / P_{2} \rightarrow P_{1} / P_{2}$ given by

$$
f_{P}([x]):= \begin{cases}{[f(x)]} & \text { if } f(x) \in P_{1} \\ {\left[P_{2}\right]} & \text { otherwise. }\end{cases}
$$

The following two theorems let us define the Conley index in the discrete case.

THEOREM 5.3 ([9]). Every isolating neighborhood for $f$ admits an index pair.

Theorem 5.4 ([11]). Assume $K$ is an isolated invariant set with respect to $f$. Let $N$ be an isolating neighborhood for $K$ and $P$ an index pair in $N$. Then the Szymczak class of $\left(P_{*},\left[f_{P}\right]\right)$ in $\operatorname{Szym}\left(\mathrm{HtpTop}_{*}\right)$ does not depend on the particular choice of $N$ and $P$.

Now we define the Conley index of $K$ by

$$
h(K, f):=h(K):=\left[P_{*},\left[f_{P}\right]\right]_{\text {Szym }}
$$

for an arbitrarily chosen index pair $P$ for $K$.

6. The monodromy class of the Conley index over $\mathbb{S}^{1}$ and the Conley index of the Poincaré map. Let $\mathbf{U}$ and $\mathbf{V}$ be semibundles with semiflows $\phi$ and $\psi$, respectively.

Proposition 6.1. Assume $\alpha: \mathbf{U} \rightarrow \mathbf{V}$ is an f.d. map. Then $\alpha \circ \Phi$ and $\Psi \circ \alpha$ are homotopic in Fib. Moreover, if $\alpha$ is a fiberwise pointed map then for any $a \in \mathbb{S}^{1}$ the maps $\alpha_{a} \circ \Phi_{a}$ and $\Psi_{a} \circ \alpha_{a}$ are homotopic in $\mathrm{Top}_{*}$ (here $\alpha_{a}$ donotes the restriction to the fiber).

Proof. One easily checks that the required homotopies are

$$
F: U \times[0,1] \ni(x, t) \rightarrow \psi(\alpha(\varphi(x, t)), 1-t) \in V
$$

and

$$
G: U_{a} \times[0,1] \ni(x, t) \rightarrow \psi\left(\alpha_{a+t}(\varphi(x, t)), 1-t\right) \in V_{a} .
$$

Proposition 6.2. For any $a, b \in \mathbb{S}^{1}$ the pairs $\left(U_{a},\left[\Phi_{a}\right]\right),\left(U_{b},\left[\Phi_{b}\right]\right)$ are isomorphic in $\operatorname{Szym}\left(\operatorname{HtpTop}_{*}\right)$.

Proof. Actually, the proposition is true even for the pairs $\left(U_{a}, \Phi_{a}\right)$ and $\left(U_{b}, \Phi_{b}\right)$ in $\operatorname{Szym}\left(\operatorname{Top}_{*}\right)$. It is obvious if $a=b$, thus assume that $a \neq b$. Let $t \in(0,1)$ be such that $b=e^{2 \pi i t} a$. Define $\Phi_{b a}: U_{a} \ni x \rightarrow \varphi(x, t) \in U_{b}$ and $\Phi_{a b}: U_{b} \ni x \rightarrow \varphi(x, 1-t) \in U_{a}$. Then $\Phi_{a}=\Phi_{b a} \circ \Phi_{a b}$ and $\Phi_{b}=\Phi_{a b} \circ \Phi_{b a}$ and the assertion follows immediately from Theorem 5.2. 
The Szymczak class of $\left(U_{a},\left[\Phi_{a}\right]\right)$ in $\operatorname{Szym}\left(\operatorname{HtpTop}_{*}\right)$ does not depend on $a \in \mathbb{S}^{1}$. By Proposition 4.1, it is also independent of the choice of $\Phi$ as the time-one map of some $r_{U}$-preserving semiflow. It will be called the monodromy class of $\mathbf{U}$ and denoted by $M(\mathbf{U})$.

A crucial role is played by the following result:

TheOREM 6.3. Assume $\mathbf{U}, \mathbf{V}$ are two compact semibundles over $\mathbb{S}^{1}$. If $\mathbf{U}$ and $\mathbf{V}$ have the same fiberwise deforming homotopy type then their monodromy classes are equal.

We assume now that $\pi$ is a flow on a locally compact metric space $X$. Let $\Pi:=\pi_{1}$ be the time-one map of $\pi$. For $a \in \mathbb{S}^{1}$ we put $X_{a}:=\xi^{-1}(a)$ and $\Pi_{a}: X_{a} \ni x \rightarrow \Pi(x) \in X_{a}$. Recall that the map $\Pi_{a}$ is called the Poincaré map.

TheOREM 6.4. Any isolated invariant set $S$ for $\pi$ admits an isolating neighborhood $N$ and a regular index pair $P$ in $N$ such that the pair $Q:=X_{a} \cap P$ is an index pair in $N_{a}:=X_{a} \cap N$ for $\Pi_{a}$.

Let $N$ be an isolating neighborhood for $\pi$ and let $P$ be a regular index pair in $N$. For $[x, i] \in U_{\xi}(P)$ and $t \in \mathbb{R}^{+}$put

$$
\pi^{P}([x, i], t):= \begin{cases}{\left[e^{2 \pi i t} x, 0\right]} & \text { if } i=0 \\ {\left[e^{2 \pi i t} \xi(x), 0\right]} & \text { if } i=1 \text { and } t \geq \sigma_{\operatorname{cl}\left(P_{1} \backslash P_{2}\right)}(x) \\ {[\pi(x, t), 1]} & \text { if } i=1 \text { and } t \leq \sigma_{\operatorname{cl}\left(P_{1} \backslash P_{2}\right)}(x)\end{cases}
$$

One easily verifies the following proposition.

Proposition 6.5. $\pi^{P}$ given by formula (6) is an $r_{U_{\xi}(P)}$-preserving semiflow on $\mathbf{U}_{\xi}(P)$.

We call it a semiflow on $\mathbf{U}_{\xi}(P)$ induced by $\pi$. The semiflow turns $\mathbf{U}_{\xi}(P)$ into a semibundle.

THEOREM 6.6. The monodromy class of $\mathbf{U}_{\xi}(P)$ does not depend on the choice of a particular regular index pair $P$ for a given isolated invariant set $S$.

Proof. Let $P$ and $Q$ be two regular index pairs for $S$. By Theorem 6.3 in $[7] \mathbf{U}_{\xi}(P)$ and $\mathbf{U}_{\xi}(Q)$ have the same fiberwise deforming homotopy type. Moreover, the spaces $U_{\xi}(P)$ and $U_{\xi}(Q)$ are compact. Thus the conclusion follows from Theorem 6.3.

The above theorem lets us introduce the notation

$$
M\left(h_{\xi}(S, \pi)\right):=M\left(\mathbf{U}_{\xi}(P)\right)
$$

for any regular index pair of $S$.

TheOREM 6.7. For any isolated invariant set $S$ with respect to $\pi$ we have

$$
M\left(h_{\xi}(S, \pi)\right)=h\left(S_{a}, \Pi_{a}\right)
$$

for any $a \in \mathbb{S}^{1}$.

7. Suspension and the discrete Conley index. Let $X$ be a locally compact space and let $f: X \rightarrow X$ be a proper map of a pointed topological space into itself. The suspension of $f$ is a topological space $\Sigma_{f}$ defined by $\Sigma_{f}:=X \times \mathbb{R}^{+} / \equiv$, where $\equiv$ is the 
smallest equivalence relation such that for any $x \in X$ and any $\tau \in \mathbb{R}^{+}$

$$
(x, \tau+1) \equiv(f(x), \tau) .
$$

(Equivalently, $\Sigma_{f}$ can be defined as the quotient space of $X \times[0,1]$ by the relation identifying $(x, 1)$ with $(f(x), 0)$. In the sequel we will also use that approach.) There is an associated projection $r_{f}: \Sigma_{f} \ni[x, b] \rightarrow e^{2 \pi i b} \in \mathbb{S}^{1}$ and an $r_{f}$-preserving semiflow $\varphi_{f}: \Sigma_{f} \times \mathbb{R}^{+} \rightarrow \Sigma_{f}$ given by

$$
\varphi_{f}([x, \tau], t):=[x, \tau+t] .
$$

If $f$ preserves a point $x_{0} \in X$, i.e. $f$ is a map of the pointed space $\left(X, x_{0}\right)$ into itself, then there is a natural embedding $s_{f}: \mathbb{S}^{1} \ni e^{2 \pi i a} \rightarrow\left[x_{0}, a\right] \in \Sigma_{f}$, where $a \geq 0$. This makes $\left(\Sigma_{f}, r_{f}, s_{f}\right)$ a fiberwise pointed space over $\mathbb{S}^{1}$ and consequently $\left(\Sigma_{f}, r_{f}, s_{f}\right)$ is a semibundle. The set

$$
\Sigma_{f}^{0}:=\left\{\left[x_{0}, b\right] \mid b \in[0,1]\right\}
$$

is obviously invariant under $\varphi_{f}$.

For $A \subset X$ put

$$
\Sigma_{f}(A):=\{[x, b] \mid x \in A, b \in[0,1]\} .
$$

Let $K$ be an isolated invariant set for $f$. The set $\Sigma_{f}(K)$ is an isolated invariant set of $\varphi_{f}$ hence we can consider $h_{r_{f}}\left(\Sigma_{f}(K), \varphi_{f}\right)$ as the Conley-type index of $K$. As a trivial consequence of Theorem 6.7 we get:

THEOREM 7.1. If $K$ is an isolated invariant set for the homeomorphism $f$ then

$$
h(K, f)=M\left(h_{r_{f}}\left(\Sigma_{f}(K), \varphi_{f}\right)\right) .
$$

It follows that $h_{r_{f}}\left(\Sigma_{f}(K), \varphi_{f}\right)$ delivers no less information than the discrete Conley index $h(K, f)$.

We conclude this section by a few results which facilitate calculation of the fiberwise deforming homotopy type of suspensions. Let $\left(X, x_{0}\right)$ be a well-pointed space, i.e. $X \times$ $0 \cup\left\{x_{0}\right\} \times I$ is a retract of $X \times I$.

Proposition 7.2. If $f, g:\left(X, x_{0}\right) \rightarrow\left(X, x_{0}\right), f \simeq g$ rel $x_{0}$ then

$$
\left[\Sigma_{f}\right]_{\mathbb{S}^{1}}=\left[\Sigma_{g}\right]_{\mathbb{S}^{1}} .
$$

Let $c_{x_{0}}$ denote the constant map $x \rightarrow x_{0}$. Recall that the fiberwise deforming homotopy type of the triple $\left(\mathbb{S}^{1}, \mathrm{id}_{\mathbb{S}^{1}}, \mathrm{id}_{\mathbb{S}^{1}}\right)$ is called trivial.

Proposition 7.3. $\Sigma_{c_{x_{0}}}$ has the trivial fiberwise deforming homotopy type over $\mathbb{S}^{1}$.

Proposition 7.4. Let $x_{0} \in A \subset X$. Assume that $A$ is a strong deformation retract of $X$. Then

$$
\left[\Sigma_{f}\right]_{\mathbb{S} 1}=\left[\Sigma_{r \circ f \circ i}\right]_{\mathbb{S}^{1}}
$$

where $r: X \rightarrow A$ is a strong deformation retraction and $i: A \hookrightarrow X$ is the inclusion.

8. Examples. Let $f: X \rightarrow X$ be a homeomorphism. Our aim is to present a possible way of calculation of the index $h_{r_{f}}\left(\Sigma_{f}(K), \varphi_{f}\right)$ (see Section 7 ) for some isolated invariant set $K$ for $f$. Recall that the definition of isolating block was given in Section 3. Note that if $B$ is an isolating block with the exit set $B^{-}$then $\left(B / B^{-},\left[B^{-}\right]\right)$is a well-pointed space 
(see [7, Prop. 6.1]). For a reason which will be made clear in the proof of the following theorem, we impose an additional condition for isolating blocks considered in the sequel. An isolating block $B$ is called proper if there exists a $\delta>0$ such that $x \cdot[-\delta, 0) \subset X \backslash B$ for every $x \in B^{+}$(or, equivalently, $x .(0, \delta] \subset X \backslash B$ for every $x \in B^{-}$).
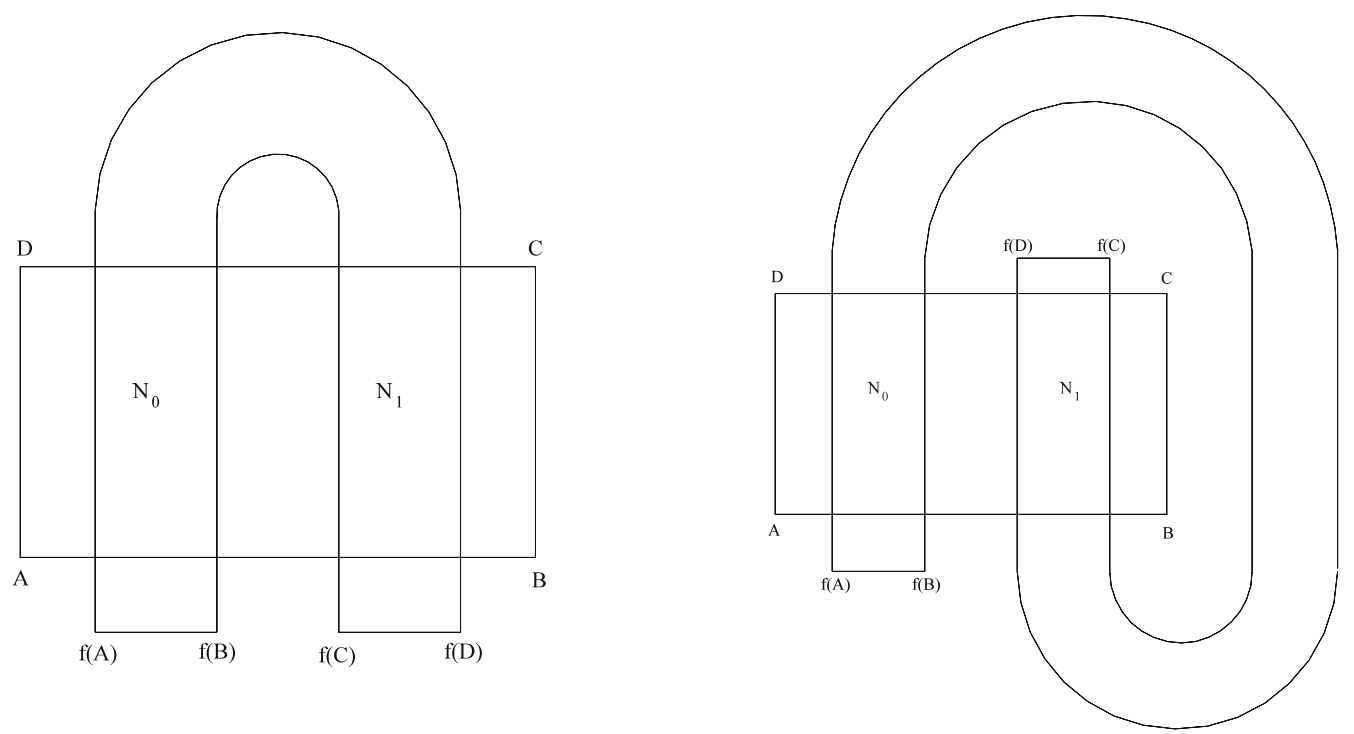

Fig. 1. $U$ - and $G$-horseshoe

THEOREM 8.1. Let $B$ be a proper isolating block for some (continuous-time) flow on $X, B^{+}$and $B^{-}$denote its entrance and exit sets, respectively. If

$$
f(B) \cap B^{+}=\emptyset, \quad f\left(B^{-}\right) \cap B=\emptyset
$$

then $K:=\left\{x \in X: f^{n}(x) \in B \forall n \in \mathbb{Z}\right\}$ is an isolated invariant set for $f$ and

$$
h_{r_{f}}\left(\Sigma_{f}(K), \varphi_{f}\right)=\left[\Sigma_{f_{\#}}\right]_{\mathbb{S}^{1}},
$$

where $f_{\#}:\left(B / B^{-},\left[B^{-}\right]\right) \rightarrow\left(B / B^{-},\left[B^{-}\right]\right)$is given by

$$
f_{\#}([x]):=\left\{\begin{array}{rll}
{[f(x)]} & \text { if } & f(x) \in B, \\
{\left[B^{-}\right]} & \text {if } & f(x) \notin B .
\end{array}\right.
$$

REMARK 8.2. The reader familiar with [12] will easily notice that under the assumptions of Theorem 8.1 the pair $\left(B, B^{-}\right)$is an index pair in the sense of [12] for the discrete dynamical system generated by the homeomorphism $f$ and $f_{\#}$ is the associated index map (cf. also [10]).

The assumption on an isolating block structure of the set $B$ in Theorem 8.1 is satisfied in reasonable situations as the following result shows:

TheOREM 8.3. Let $X$ be a smooth manifold (without boundary) and $B$ be a $C^{1}$-class compact submanifold-with-corners of $X, \operatorname{dim} B=\operatorname{dim} X$, such that

$$
\partial B=B_{1} \cup B_{2},
$$


where $B_{1}$ and $B_{2}$ are codimension-one $C^{1}$-class submanifolds-with-boundary of $X$,

$$
\partial B_{1}=\partial B_{2}=B_{1} \cap B_{2} .
$$

Then there exists a flow on $X$ such that $B$ is its proper isolating block, $B^{+}=B_{1}$ and $B^{-}=B_{2}$. In particular, if (7) is satisfied then the conclusion of Theorem 8.1 holds.

As examples of the computation of the Conley index over $\mathbb{S}^{1}$ we study the suspension flows of the $G$ - and $U$-horseshoes.

Let us recall that the $G$ - and $U$-horseshoes are homeomorphisms of the plane which transform a rectangle to the shape of $U$ and $G$, respectively, as depicted in Figure 1. The rectangle $B:=\mathbf{A B C D}$ is a submanifold-with-corners, its boundary is the union of $B_{1}$ and $B_{2}$ where $B_{1}:=\mathbf{A D} \cup \mathbf{B C}$, the union of the sides, and $B_{2}:=\mathbf{A B} \cup \mathbf{C D}$, the union of the bases. By Theorem 8.3, $B$ is a proper isolating block for some planar flow, $B^{+}=B_{1}$ and $B^{-}=B_{2}$. It is clear from the figure that the condition (7) is satisfied, hence, by Theorem 8.1, in both cases the index $h_{r_{f}}\left(\Sigma_{f}(K), \pi_{f}\right)$ of the suspension of the isolated invariant set $K$ determined by a horseshoe $f$ is equal to the fiberwise deforming homotopy type of $\Sigma_{f_{\#}}$.

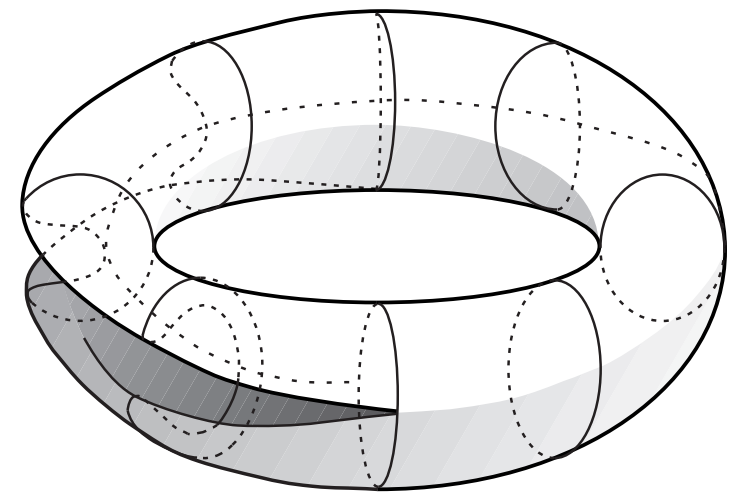

Fig. 2. The Conley index over $\mathbb{S}^{1}$ of the suspension of the $U$-horseshoe

After collapsing $B / B_{1}$ to a circle, by Proposition 7.4, the latter type can be expressed as $\left[\Sigma_{D}\right]_{\mathbb{S}^{1}}$ in the case of $U$-horseshoe and as $\left[\Sigma_{E}\right]_{\mathbb{S}^{1}}$ in the case of $G$-horseshoe, where $D, E:\left(\mathbb{S}^{1}, 1\right) \rightarrow\left(\mathbb{S}^{1}, 1\right)$ are defined by

$$
D\left(e^{2 \pi i t}\right):=\left\{\begin{aligned}
e^{4 \pi i t} & \text { if } \quad t \in\left[0, \frac{1}{2}\right] \\
e^{4 \pi i(1-t)} & \text { if } \quad t \in\left[\frac{1}{2}, 1\right]
\end{aligned}\right.
$$

and by $E(z):=z^{2}$. Since $\left(\mathbb{S}^{1}, 1\right)$ is a well-pointed space and $D$ is homotopic to the constant map $c_{1}$, one easily concludes from Propositions 7.2 and 7.3 that the Conley index over $\mathbb{S}^{1}$ of the suspension of the isolated invariant set determined by the $U$-horseshoe is trivial.

The visualization of the sample semibundle representing the Conley index over $\mathbb{S}^{1}$ of the suspension of the $U$-horseshoe is presented in Figure 2 and of the suspension of the $G$-horseshoe in Figure 3 . 


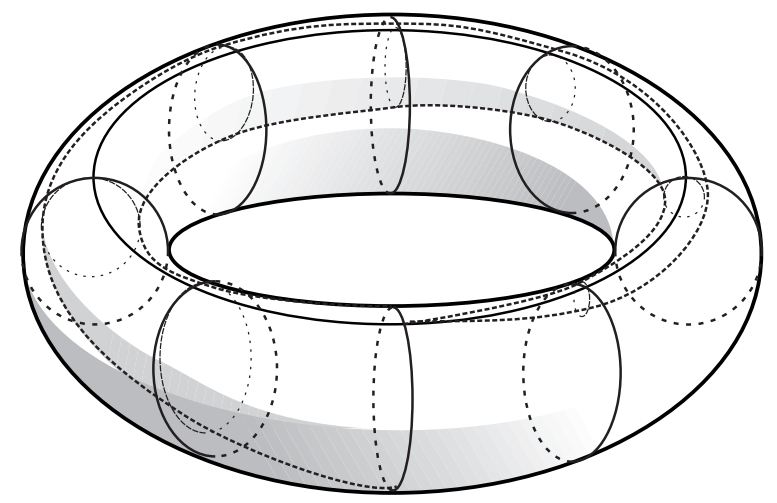

Fig. 3. The Conley index over $\mathbb{S}^{1}$ of the suspension of the $G$-horseshoe

\section{References}

[Bo] K. Borsuk, Theory of Retracts, PWN, Warszawa, 1967.

[1] I. M. James, General topology over a base, in: I. M. James, E. H. Kronheimer (editors), Aspects of Topology, Cambridge University Press, Cambridge, 1985.

[2] I. M. James, General Topology and Homotopy Theory, Springer-Verlag, New York, 1984.

[3] I. M. James, Fibrewise Topology, Cambridge University Press, Cambridge, 1989.

[4] T. KaCZynski and M. MrozeK, Connected simple systems and the Conley functor, Topol. Methods Nonlinear Anal. 10 (1997), 183-193.

[5] Ch. McCord, K. Mischaikow, and M. Mrozek, Zeta functions, periodic trajectories and the Conley index, J. Differential Equations 121 (1995), 258-292.

[6] M. Mrozek, Leray functor and cohomological index for discrete dynamical systems, Trans. Amer. Math. Soc. 318 (1990), 149-178.

[7] M. Mrozek, J. F. Reineck, and R. Srzednicki, The Conley index over a base, Trans. Amer. Math. Soc., to appear.

[8] M. Mrozek, J. F. Reineck, and R. Srzednicki, The Conley index over the circle, J. Dynamics Differential Equations, to appear.

[9] M. Mrozek and K. P. Rybakowski, A cohomological Conley index for maps on metric spaces, J. Differential Equations 90 (1991), 143-171.

[10] J. W. Robbin and D. Salamon, Dynamical systems, shape theory and the Conley index, Ergodic Theory Dynamical Systems 8* (1988), 375-393.

[11] A. Szymczak, The Conley index for discrete semidynamical systems, Top. \& Appl. 66 (1995), 215-240.

[12] A. Szymczak, A combinatorial procedure for finding isolating neighborhoods and index pairs, Proc. Royal Soc. Edinburgh Sect. A 127 (1997). 